\title{
EXPERIMENTAL METHOD FOR PLOTTING S-N CURVE WITH A SMALL NUMBER OF SPECIMENS
}

\author{
Przemysław Strzelecki, PhD \\ Janusz Sempruch, Prof. \\ University of Science and Technology in Bydgoszcz, Poland
}

\begin{abstract}
The study presents two approaches to plotting an $S$-N curve based on the experimental results. The first approach is commonly used by researchers and presented in detail in many studies and standard documents. The model uses a linear regression whose parameters are estimated by using the least squares method. A staircase method is used for an unlimited fatigue life criterion. The second model combines the $S$ - $N$ curve defined as a straight line and the record of random occurrence of the fatigue limit. A maximum likelihood method is used to estimate the S-N curve parameters. Fatigue data for $C 45+C$ steel obtained in the torsional bending test were used to compare the estimated S-N curves. For pseudo-random numbers generated by using the Mersenne Twister algorithm, the estimated S-N curve for 10 experimental results plotted by using the second model, estimates the fatigue life in the scatter band of the factor 3. The result gives good approximation, especially regarding the time required to plot the $S$ - $N$ curve.
\end{abstract}

Keywords: high-cycles fatigue, S-N curve, fatigue tests, number of specimens

\section{INTRODUCTION}

To determine a fatigue life or fatigue limit of structural components, the inter-dependencies resulting from the material data of one of the S-N curves, are used. The S-N curves are used in calculations for both limited and unlimited fatigue life regimes.

For the limited fatigue life regime, it is most often represented by the coefficient $m$ defined by the following equation:

$$
\log (\mathrm{N})=\mathrm{m} \log (\mathrm{S})+\mathrm{c}
$$

where $N$ is the number of load cycles, $S$ is a stress amplitude, and $c$ is an absolute constant term in the expression. The recommendations on selecting value of the coefficient $m$ are available in the literature. A critical analysis of the recommended values is shown in Tab.1, based on a set of values of the coefficient $m$ taken from the literature (based on 91 material data sets for steel). Fig. 1 shows the histogram for the available material data. The average recommended values are considered accurate although significant deviations can be observed in some cases. The deviations are related to a large scatter of the coefficient $m$. Based on the estimated gamma distribution (shown in Fig. 1) a $(9.51 \div 15.26)$ scatter can be observed for $50 \%$ probability. For $95 \%$ probability, the scatter is greater and amounts to $5.61 \div 22.51$, which can pose serious problems with accurate determination of a correct value.

A complete $\mathrm{S}-\mathrm{N}$ curve can be plotted by using an analytical method. Selected methods $[1,2,3,4,5]$ were verified by the 
authors of this study. Fig. 2 shows the histogram of error distribution of fatigue life estimation for both methods. Sign "+" indicates that the estimated values are on the safe side. Sign "-" indicates the opposite. In case the curve shifts from safe to unsafe side, " $+/-$ " sign is assigned. The opposite is marked with "/+" sign. Based on the results of Fig. 2 the practical implementation of the analytical methods does not only affect modelling accuracy but also the quality of determining the position of the estimated fatigue life in relation to the actual fatigue life. The methods may result in over-estimation or under-estimation of the fatigue life, which is very difficult to anticipate a priori.

Tab. 1 Guidelines for selection of recommended number of specimens

\begin{tabular}{|c|c|}
\hline Value of the coefficient $m$ & Probability of getting $m$-value \\
\hline $5 \div 20$ acc. $[1]$ & $92,6 \%$ \\
\hline $6 \div 20$ acc. $[2]$ & $90,4 \%$ \\
\hline $8 \div 12$ acc. [3] & $35,3 \%$ \\
\hline $5,61 \div 22,51$ & $\begin{array}{l}\text { 95\% (estimated from histogram of } \\
\text { Fig. 1) }\end{array}$ \\
\hline $9,51 \div 15,26$ & $\begin{array}{c}50 \% \text { (estimated from the histogram } \\
\text { of Fig. 1) }\end{array}$ \\
\hline
\end{tabular}

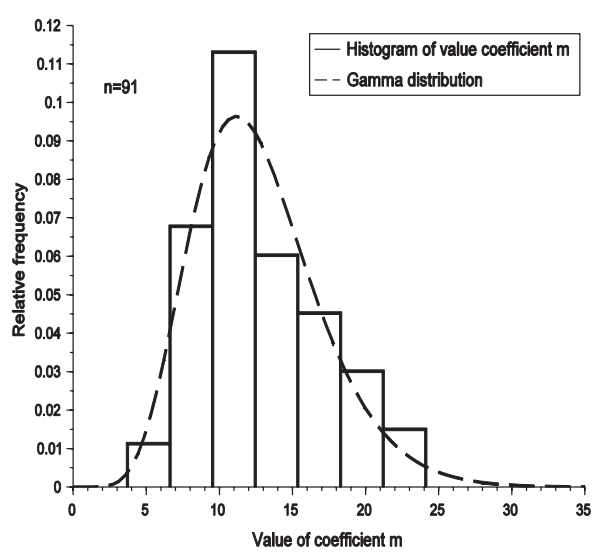

Fig. 1. Distribution of the coefficient $m$ for smooth specimen of constructional steel under normal stress

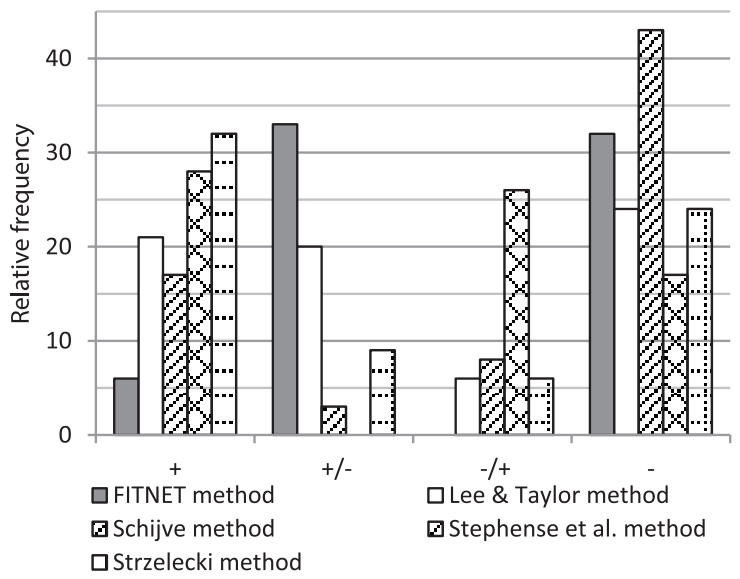

Fig. 2. Distribution of the coefficient $m$ for smooth specimen of constructional steel under normal stress
Although there is an average characteristic error for the method, see Fig. 3 , the scatter of error still remains significant, and to determine the correction factor (as a safety factor) to reduce the estimation inaccuracy, is still difficult.

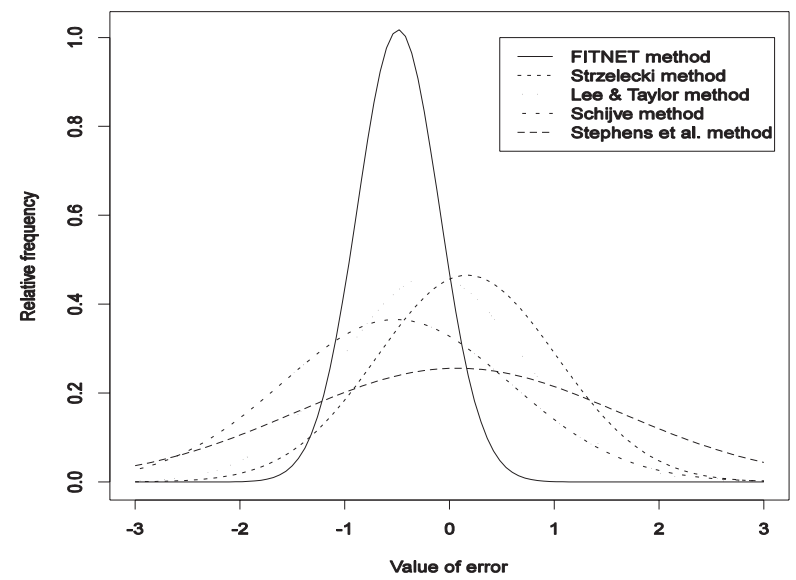

Fig. 3. Distribution of the coefficient $m$ for smooth specimen of constructional steel under normal stress

The study [8] discusses that along with the strictly experimental methods (considered accurate) and the analytical methods (burdened with uncertainty as shown below) a hybrid solution is available by combining the available knowledge on analytical and experimental methods. Similar approach is discussed in study [9]. Fig. 4 shows an example of how in the fatigue tests the amount of work spent for experimental support of the analytical method, affects the error of fatigue life estimation. The points marked with numbers in Fig. 4 correspond to the following values: 1 - analytical method error where material values are determined by using tensile test, 2 - analytical/experimental method error determined by using tensile test and fatigue test for three specimens at 105 load cycles, 3 - analytical/experimental method error determined by using tensile test and the fatigue limit Zgo by using Locati method, 4 - scatter of results obtained by using the method described in [10] at 95\% confidence level.

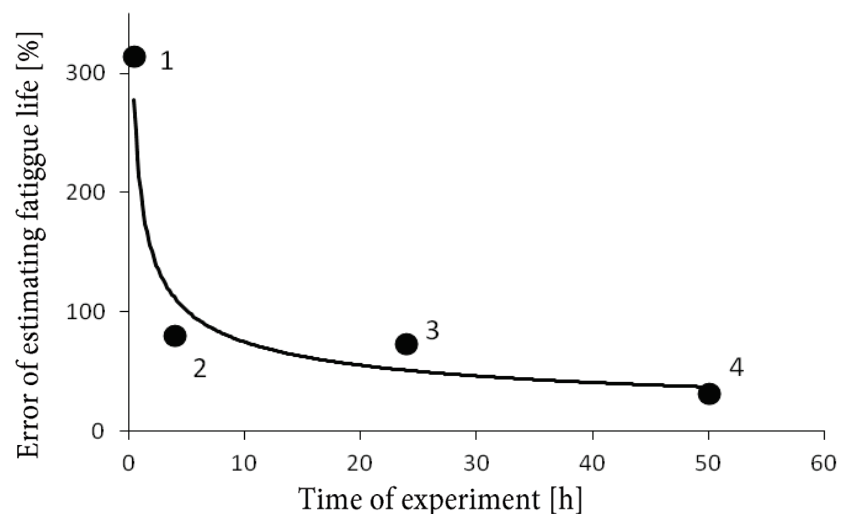

Fig. 4. Diagram of value of fatigue life estimating error according to time of carrying out the test [7] - a proposal of idea

Results for experimental verification of the approach were obtained at the torsional bending test stand shown in Fig. 5 
(described and verified in [11]). Based on the graph shown in Fig. 5 the hybrid approach is preferred to determine the fatigue life of a notched specimen considered to be a model of a structural component.

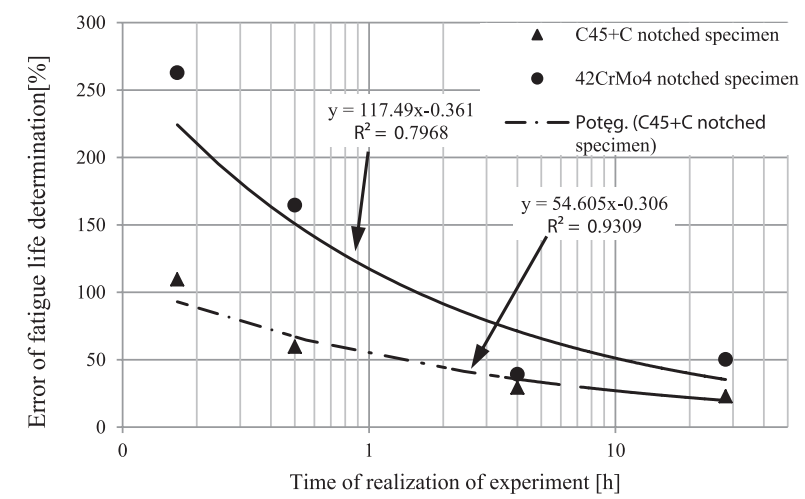

Fig. 5. The analytical method error versus the number of experimental values [8] - verification of idea

Only experimental tests yield results with an anticipated probability of the correct value estimation. Costly (due to the required time) fatigue tests can be significantly reduced with the new concepts.

The purpose of the study is to determine the fatigue life and fatigue limit modelling process for limited and unlimited fatigue life regime by experimental plotting the $\mathrm{S}-\mathrm{N}$ curve with a small number of specimens.

\section{S-N CURVE ESTIMATION METHODS}

Fig. 6 shows an estimation diagram for S-N curve based on the experimental data. The most common method to describe the relationship between the fatigue life and the load amplitude for a limited fatigue life regime is a linear regression, as given by Eq. (1). The equation parameters are commonly determined by using the least squares method described in detail in [12]. The equations to estimate the parameters of Eq.(1) are as follows:

$$
\begin{gathered}
\widehat{m}=\frac{\sum_{i=1}^{k}\left(X_{i}-\bar{X}\right)\left(Y_{i}-\bar{Y}\right)}{\sum_{i=1}^{k}\left(X_{i}-\bar{X}\right)^{2}}, \\
\hat{c}=\bar{Y}-\widehat{m} \bar{X},
\end{gathered}
$$

where $\mathrm{Y}$ is $\log (\mathrm{N}), \mathrm{X}$ is $\log (\mathrm{S})$, and $\mathrm{k}$ is the sample size. $\hat{c}$ stands for an estimated parameter, and $\bar{m}$ stands for an average value. Standard deviation is calculated by means of the following formula,[12]:

$$
\sigma=\sqrt{\frac{\sum_{i=1}^{k}\left(Y_{i}-\hat{Y}\right)^{2}}{k-2}} .
$$

Standard requirements define various numbers of specimens. 15 specimens at 5 load levels are recommended as a minimum, by [10]. Study [13] recommends to initially determine the S-N curve for 5 specimens, one for each load level and further tests to increase the S-N curve accuracy, although it does not specify a recommended number of specimens. Another study [14] recommends to determine the S-N curve for at least 28 specimens. For preliminary tests, it recommends to carry out 7 tests, but it does not specify a required number of load levels. It only includes a reference to [12] where the relation to replication percentage is defined:

$$
P R=100\left[1-\left(\frac{S l}{n_{a}}\right)\right][\%]
$$

where $S l$ is the number of load levels and $n_{a}$ is a total number of specimens.

The standard recommends to achieve the replication percentage as shown in Tab. 2 .

Tab.2 Guidelines for recommended percent replication for various tests [12]

\begin{tabular}{|l|c|}
\hline \multicolumn{1}{|c|}{ Type of tests } & Percent replication \\
\hline $\begin{array}{l}\text { Preliminary and exploratory (research and } \\
\text { development tests) }\end{array}$ & 17 to 33 \\
\hline $\begin{array}{l}\text { Research and development testing of } \\
\text { components and specimens }\end{array}$ & 33 to 50 \\
\hline Design allowable data & 50 to 75 \\
\hline Reliability data & 75 to 88 \\
\hline
\end{tabular}

When determining fatigue limit for unlimited fatigue life regime, a staircase method is often used based on Dixon and Mood concept discussed in $[3,5]$. The method requires a minimum of 15 specimens. The staircase method consists in tests at constant load, constant load frequency and uniform temperature. The number of load cycles is measured until failure or until a specified number of cycles $N_{G}$ (e.g. 5.106) is reached. A limit number of cycles is selected based on the type of used material. For the initial specimen stress amplitude is selected below the anticipated fatigue limit. For the subsequent specimen the load is reduced following the failure of the initial specimen. Otherwise (without specimen failure, or when the specimen reaches the limit number of cycles) the load is increased. The difference between the load levels are determined lower than or equal to $0,05 \cdot Z_{G}$. Example test procedure using the staircase method is shown in Fig. 6 in the bottom left corner.

Fatigue limit is calculated by means of the following formula $[3,5]$ :

$$
Z_{G}=S_{0}+S_{i}\left(\frac{A}{F} \pm \frac{1}{2}\right)
$$

where: $\mathrm{S}_{0}$ is an initial stress amplitude,

$S_{i}$ - the stress amplitude between subsequent load levels, $F$ - a total value of less frequent events,

A is defined as:

$$
A=\sum_{i=1}^{n} i \cdot f
$$


where $i$ is the ordinal number of the load level, for $S_{0}$ it is 0 , and $f$ is the number of less frequent events at the $i$-th level, and $n$ is the total number of load levels. Sign "+" in Eq. (7) indicates that the more frequent event is specimen failure, whereas “.”" indicates that the failure is a less frequent event. Standard deviation is calculated as:

$$
s=1,62 \cdot \sigma_{i}\left(\frac{F B-A^{2}}{F^{2}}+0,029\right),
$$

where:

$$
B=\sum_{i=1}^{n} i^{2} \cdot f
$$

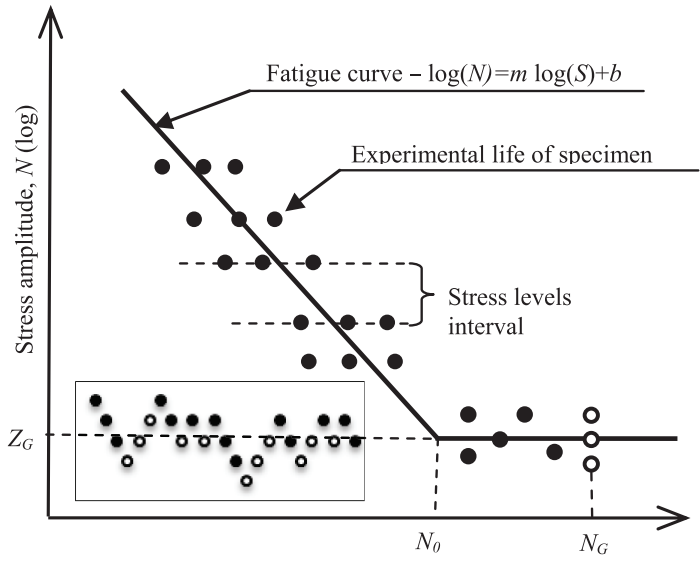

Number of cycles, $N(\log )$

Fig. 6. Scheme of estimated fatigue curve in high cycle range and fatigue limit

Plotting the S-N curve by using linear regression and staircase method is referred to as the classic approach and the first model ( Model I)

Based on the assumptions presented in [15], an alternative approach is referred to as the second model ( Model II), presented below. The advantage of the second model is the ability to plot the complete $\mathrm{S}-\mathrm{N}$ curve with a smaller number of specimens. The basis for the procedure is as follows: Eq. (1) is expressed as:

$$
\log (N)=m \log (S)+c+\varepsilon_{n}
$$

where $\varepsilon_{n}$ is a random component of fatigue life with normal distribution. The density function can be expressed as:

$$
f(N)=\frac{1}{\sqrt{2 \pi \sigma_{n}^{2}}} \exp \left(\frac{(\log (N)-(m \log (S)+b))^{2}}{\sigma_{n}^{2}}\right)
$$

where $\sigma_{n}$ is standard deviation.

The fatigue limit can be expressed as:

$$
S_{i}=Z_{G}+\varepsilon_{S}
$$

where $\varepsilon_{s}$ is a random component of fatigue limit with normal distribution. The density function can be expressed in this case as follows:

$$
f(S)=\frac{1}{\sqrt{2 \pi \sigma_{S}^{2}}} \exp \left(\frac{\left(S-Z_{G}\right)^{2}}{\sigma_{S}^{2}}\right)
$$

where $\sigma_{s}$ is standard deviation.

It can be assumed that the failure can occur as a result of a stress higher that the limit value $Z$ or after the fatigue life higher than or equal to that defined by Eq. (10), is reached. Since the values are random it can be assumed that the probability of failure will be equal to the probability of value satisfying Eq. (11) and (13). With this assumption, the probability of failure for limited and unlimited fatigue life regime can be expressed as follows, [16]:

$$
\begin{aligned}
& P\left(N<N_{i}\right)= \\
& \Phi\left(\frac{\log N_{i}-\left(a \cdot \log S_{i}+b\right)}{\sigma_{v}}\right) \cdot \Phi\left(\frac{S_{i}-Z_{s}}{\sigma_{s}}\right) \\
& \quad=q
\end{aligned}
$$

where $\Phi$ is the normal distribution function and $\mathrm{q}$ is the probability of specimen failure.

The maximum likelihood method is used to determine the parameters in Eq. (14). The method can be applied to the specimens that reached the limit number of cycles. The likelihood function is expressed as follows, [15]:

$$
\begin{aligned}
L(\theta) & =\prod_{j=1}^{k}\left[f_{n}\left(N_{j} ; S_{j}, \theta\right)\right]^{\delta_{j}}[1 \\
& \left.-F_{n}\left(N_{j} ; S_{j}, \theta\right)\right]^{1-\delta_{j}},
\end{aligned}
$$

where

$$
\delta_{j}=\left\{\begin{array}{lll}
1 & \text { if } N_{i} & \text { is a failure } \\
0 & \text { if } N_{i} & \text { is a censored observation. }
\end{array}\right.
$$

The maximum likelihood function is expressed as:

$L(\theta)=\phi\left(\frac{\log N_{i}-\left(a \cdot \log s S_{i}+b\right)}{\sigma_{v}}\right)^{\delta_{i}}$.

$\Phi\left(\frac{S_{i}-S_{x e}}{\sigma_{x e}}\right)^{\delta_{i}} \cdot\left(1-\left(\Phi\left(\frac{\log N_{i}-\left(a \cdot \log S_{i}+b\right)}{\sigma_{v}}\right) \cdot \Phi\left(\frac{S_{i}-S_{x e}}{\sigma_{x e}}\right)\right)\right)^{1-\delta_{i}}$,

where $\Phi$ is the normal distribution density function.

\section{VERIFICATION OF THE METHOD}

Data from the fatigue tests of $\mathrm{S} 45+\mathrm{C}$ steel were used to verify both models. The fatigue test was carried out on the test stand as presented in [11]. Tab. 3. shows values of static properties of the tested material. The specimens were prepared from the drawn bar in as - delivered state. Fig. 7 shows the specimen geometry.

Tab. 3 Properties of C45+C material used for calculation [11]

\begin{tabular}{|c|c|}
\hline Ultimate strength Su [MPa] & 826 \\
\hline Yield stress Sy [MPa] & 647 \\
\hline Hardness HB & 232 \\
\hline
\end{tabular}


Fig. 8 shows experimental test results for $\mathrm{C} 45+\mathrm{C}$ steel with S-N curves plotted by using Model I and Model II. The tests were performed on 31 specimens in limited fatigue life regime and 19 specimens in unlimited fatigue life regime (7 specimens failed and 12 specimens reached the limit number of cycles NG). All S-N curves were plotted at $50 \%$ probability of failure. All calculations were performed by using R software, version 3.1.3, [17].

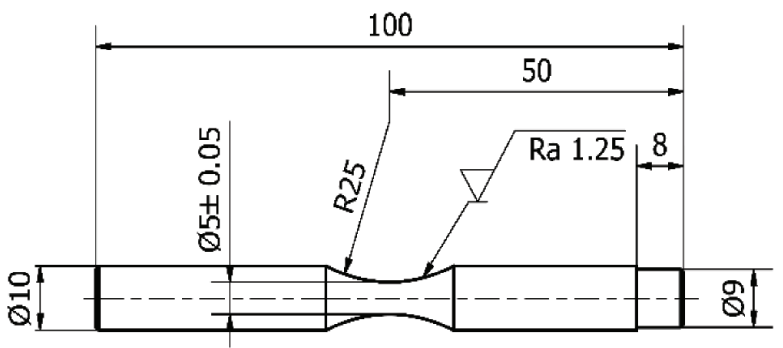

Fig. 7. Specimen used for tests

$\mathrm{C} 45+\mathrm{C}$

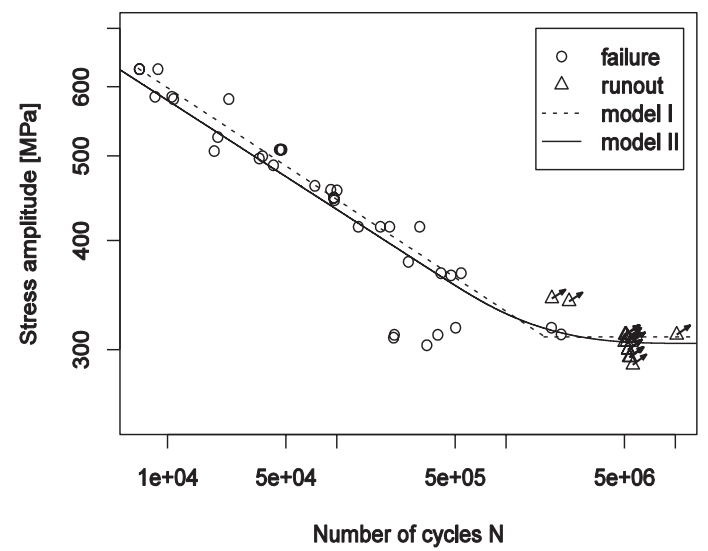

Fig. 8. Fatigue data of $\mathrm{C} 45+C$ steel and fitted fatigue characteristics by using model I and model II

The equations for Model I are shown below.

$$
\log \left(N_{i}\right)=-7.8 \log \left(S_{i}\right)+59.2 \pm 0.28
$$

$$
Z_{G}=310.3 \pm 11.4
$$

The equation according to Model II is presented by means of the formula (20).

$$
\begin{gathered}
\Phi\left(\frac{\log N_{i}-\left(-8 \cdot \log S_{i}+60.2\right)}{0.67}\right) \\
\cdot \Phi\left(\frac{S_{i}-304.9}{20}\right)=q
\end{gathered}
$$

Fig. 9 shows the results obtained by using the staircase method.

The coefficients obtained by using Model I and Model II vary to each other. The differences can be observed in the $\mathrm{S}-\mathrm{N}$ curves shown in Fig. 8. Since the S-N curve for Model II is shifted to the left resulting in the under-estimation of the fatigue life, the situation can be considered safe. Standard deviations for limited and unlimited fatigue life regimes are higher for the Model II equation (0.67 to 0.28 and 20 to 11.4, respectively) due to the higher scatter of specimen fatigue life near the fatigue limit.

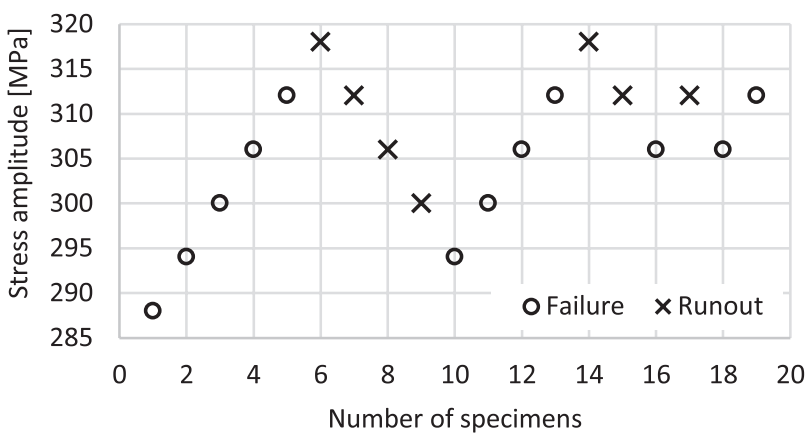

Fig. 9. Fatigue data from staircase method

\section{SMALL NUMBER OF SPECIMENS TO PLOT THE S-N CURVE}

A predefined number of specimens was used in Model I, $[9,12,13]$. No explicit number of tests was specified for Model II. How far can the number of specimens be reduced in Model II allowing for the number of specimens used in the tests based on Model I?

An existing set of 50 test results was used to verify Model II for a small number of specimens by random generating smaller subsets with the use of the following method. Ten (10) random specimens were generated. Five (5) specimens were generated for the tests using the staircase method (shown in Fig. 9) and 5 for a limited fatigue life (stress amplitude from 341 $\mathrm{MPa}$ to $628 \mathrm{MPa}$ ). The test results were divided into 5 groups by stress amplitude before generating random specimens for a limited fatigue life. One specimen was randomly selected for each range. The selected specimens are shown in Fig. 10, 11, $12,13,14$ and 15. A rectangular distribution with the density function defined by Eq. [17] was used to generate the pseudorandom numbers:

$$
f(x)=1 /(\max -\min ) .
$$

Pseudo-randomness has been achieved by using the Mersenne Twister algorithm.

Fig. 16 shows gamma distribution of the coefficient $\mathrm{m}$, previously shown in Fig. 1, as well as the modal value of the distribution and the value obtained by using Model I. A range of the obtained value for Model II for the randomly generated specimens is also included. The values of the coefficient $m$ estimated by using the maximum likelihood method were lower than 7.8 (i.e. the value estimated by using Model I), where 
the generated test results were within the unlimited fatigue life range at the number of cycles below 106. It caused the S-N curve to shift to the left. This can be observed in Fig. 10, 11,14 and 15 . It is clearly visible for the 5 th and 6 th generated random number.

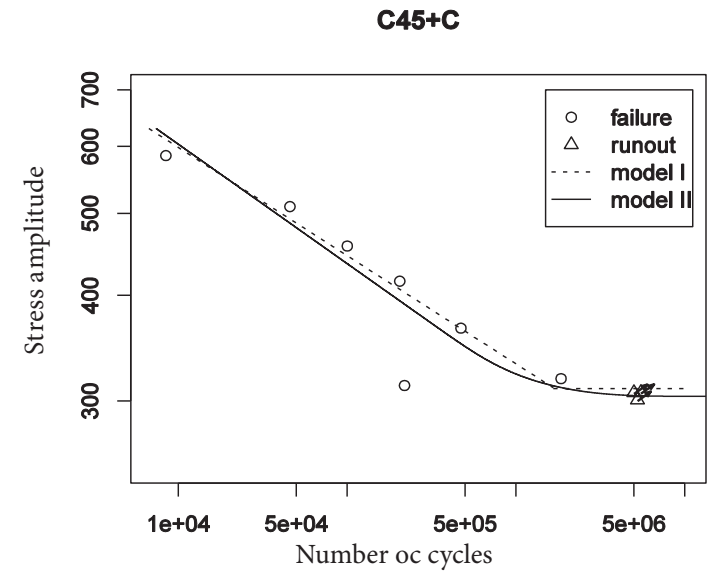

Fig. 10. Fatigue data of $\mathrm{C} 45+C$ steel from 1 st generated random number of specimens and fitted fatigue characteristics by using Model II and Model I described by Eq. (18) and (19), respectively.

C45+C

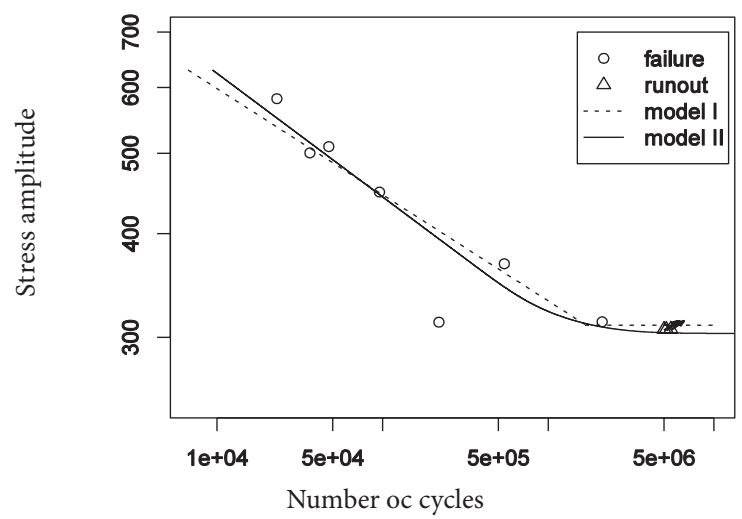

Fig. 11. Fatigue data of $C 45+C$ steel from 2 nd generated random number of specimens and fitted fatigue characteristics by using Model II and Model I described by Eq. (18) and (19), respectively.

C45+C

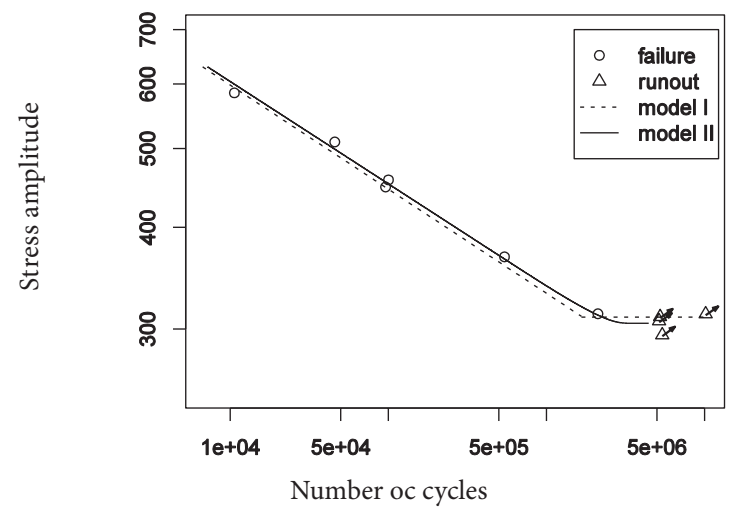

Fig. 12. Fatigue data of $C 45+C$ steel from 3 rd generated random number of specimens and fitted fatigue characteristics by using Model II and Model I described by Eq. (18) and (19), respectively.

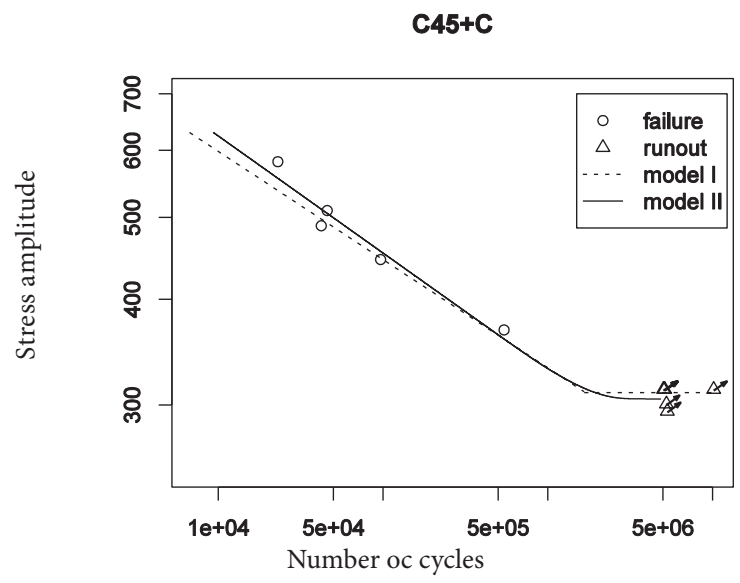

Fig. 13. Fatigue data of $C 45+C$ steel from 4 th generated random number of specimens and fitted fatigue characteristics by using Model II and Model I described by Eq. (18) and (19), respectively.

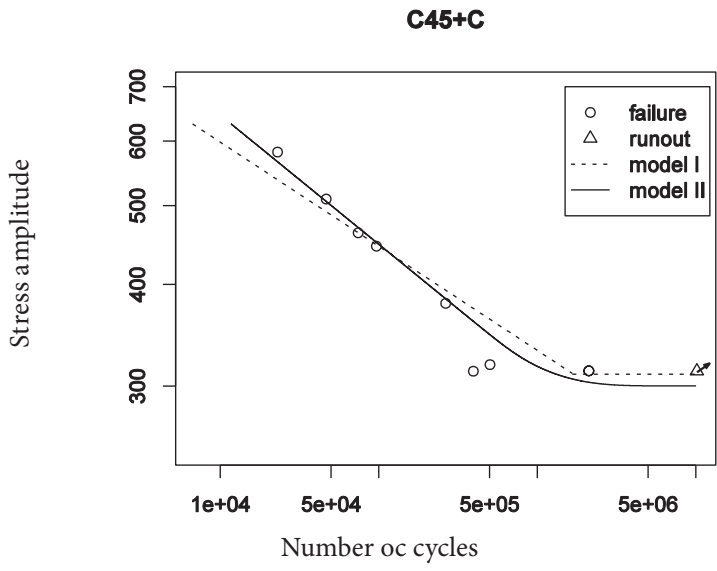

Fig. 14. Fatigue data of $\mathrm{C} 45+C$ steel from 5 th generated random number of specimens and fitted fatigue characteristics by using Model II and Model I described by Eq. (18) and (19), respectively.



Fig. 15. Fatigue data of $C 45+C$ steel from 6 th generated random number of specimens and fitted fatigue characteristics by using Model II and Model I described by Eq. (18) and (19), respectively. 
Tab. 4 Values of coefficients for Model II estimated from the data generated by psedo-random procedure

\begin{tabular}{|c|c|c|c|c|c|}
\hline No. & $a$ & $b$ & $\sigma_{v}$ & $Z_{s}$ & $\sigma_{s}$ \\
\hline $\mathbf{1}$ & -7.1 & 54.6 & 0.71 & 303.7 & 19.4 \\
\hline $\mathbf{2}$ & -6.7 & 52.4 & 0.69 & 303.3 & 19.4 \\
\hline $\mathbf{3}$ & -8 & 60.1 & 0.13 & 304.9 & 20 \\
\hline $\mathbf{4}$ & -.72 & 55.7 & 0.51 & 305 & 20 \\
\hline $\mathbf{5}$ & -6.3 & 50 & 0.51 & 301.5 & 17.4 \\
\hline $\mathbf{6}$ & -6 & 47.8 & 0.55 & 302.4 & 19.8 \\
\hline
\end{tabular}

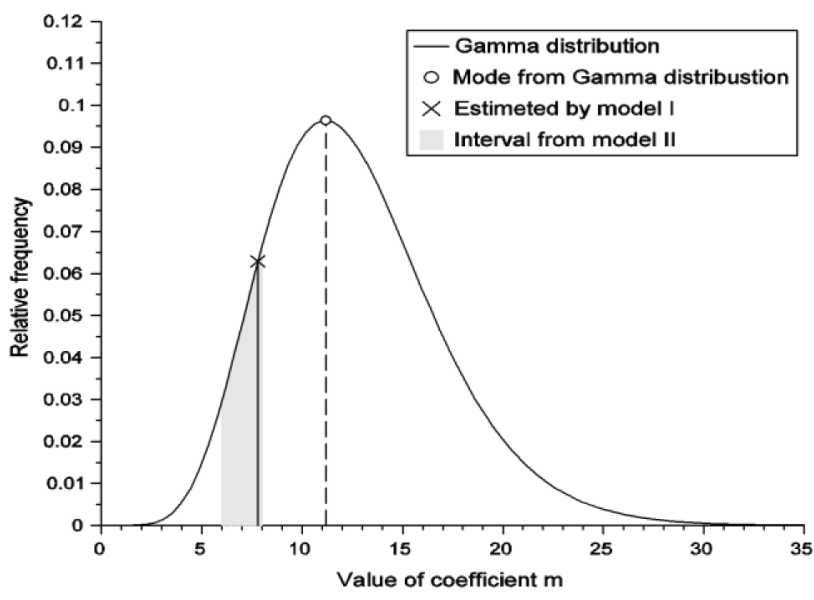

Fig. 16. Distribution of the coefficient $m$ for smooth specimen of constructional steel under normal stress (from Fig. 1) and its estimated value from Model I and II.

Fig. 17 shows comparison of the fatigue life determined by using experimental method and that estimated by using Model I. Fig. 18, Fig. 19 and Fig. 20 show comparison of fatigue life determined from S-N curve obtained by using Model I and Model II for all specimens and two randomly generated data.

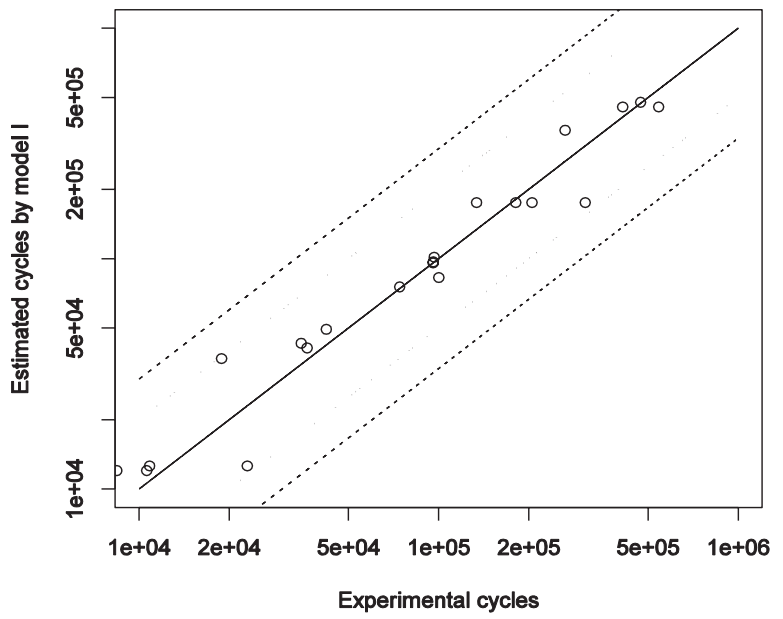

Fig. 17. Comparision of experimental value of fatigue cycles and that calculated by using Model I

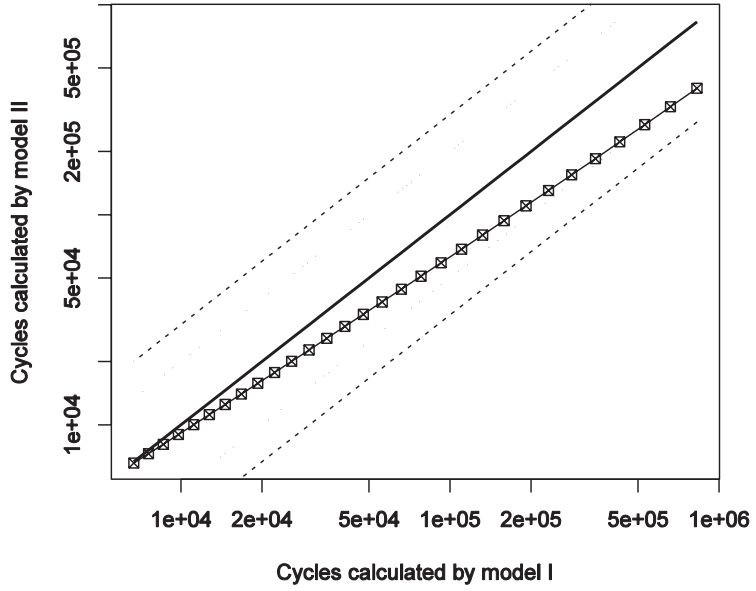

Fig. 18. Comparision of fatigue cycles calculated by using Model I and II for all specimens

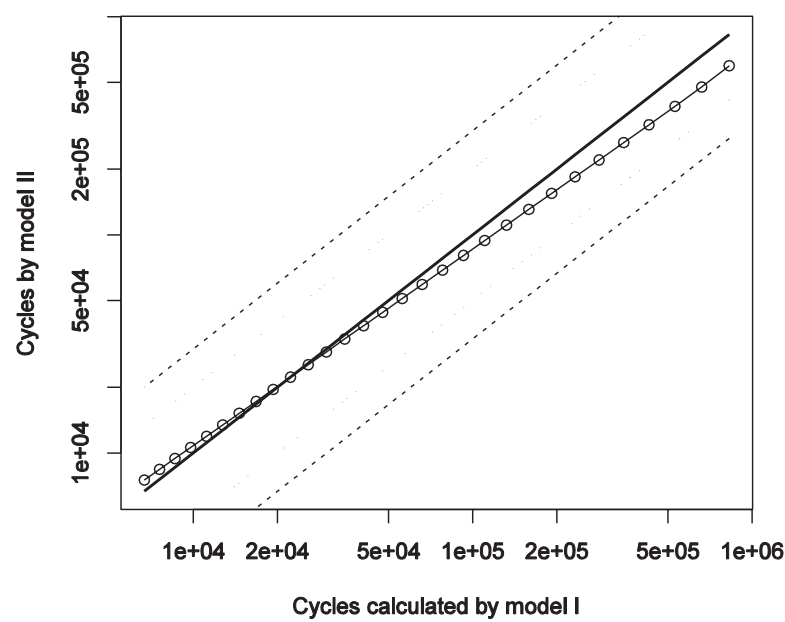

Fig. 19. Comparision of fatigue cycles calculated by using Model I and II for pseudo-random 1

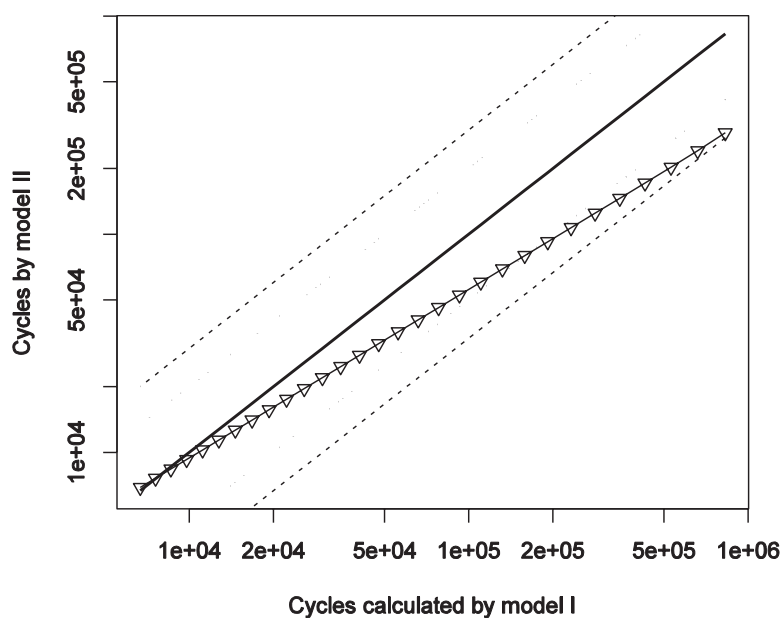

Fig. 20. Comparision of fatigue cycles calculated by model I and II for pseudorandom 6 - the least favorable generated pseudo-random

\section{ANALYSIS AND SUMMARY}

The S-N curves estimated by means of Model I and Model II are similar to each other for all specimens. The difference in the estimated fatigue life shown in Fig. 18. Fig. 18 is within 
the band 2. It means that the difference is small considering the scatter of test results compared to the fatigue life estimated from the S-N curve based on Model I (Fig. 17). For the S-N curves determined by using pseudo-random- generated data, the difference in estimated fatigue life are within the band 3 . For the estimated fatigue limit value, the difference between Model I and Model II is 5.4 MPa. The maximum difference for pseudo-random - generated data is $8.8 \mathrm{MPa}$.

The following conclusions have been drawn from the study:

1. The differences in the fatigue life estimated from the S-N curve based on Model I and Model II differ by no more than the scatter band of the experimental results.

2. The differences between the estimated fatigue limit values for Model I and II , for all results and pseudorandom results, are within the standard deviation expressed by Eq. (19).

3. Standard deviations for the fatigue limit were higher for the S-N curve estimated from Model II, which is most likely due to allowing for ? the specimens that have reached the base number of cycles.

4. The advantage of Model II compared to Model I is the ability to determine a complete $\mathrm{S}-\mathrm{N}$ curve by using the least number of specimens. The S-N curve was determined by using Model II and allowed fatigue life to be estimated with a satisfactory accuracy for 10 specimens. According to standard requirements the number of specimens is to be 30 .

5. Standard deviation values for S-N curve estimated from Model II (see Eq. (20) and Tab. 4) were, in most cases, higher than those estimated from Model I (see Eq.(18)).

6. Considering the above presented conclusions, Model II can be used to determine a complete $\mathrm{S}-\mathrm{N}$ curve when the set of experimental results contains less data than required by the standards.

7. Since the obtained results are of particular interest, similar verifications are recommended for other structural materials.

\section{BIBLIOGRAPHY}

1. I. Stephens, Ralph, A. Fatemi, R. Stephens, Robert, O. Fuchs, Henry: Metal Fatigue in Engineering (1980), John Wiley and Sons, 2001.

2. Z. Dyląg, Z. Orłoś,: Fatigue strength of materials (in Polish), Wydawnictwo Naukowe-Techniczne, 1962.

3. S. Kocańda, J. Szala: Basic metods for fatigue calculations (in Polish), (1997)

4. M. Kocak, S. Webster, J. Janosch, J., A. Ainsworth, R., R. Koers: FITNET Fitness-for-Service PROCEDURE - FINAL DRAFT MK7, 2006.

5. Y.L. Lee, J. Paw, B. Hathaway, Richard, E. Barkey, Mark: Fatigue Testing and Analysis - Theory and Practice, Elsevier Butterworth-Heinemann, 2005.
6. J. Schijve : Fatigue of structures and materials, Second Edition, Springer Science+Business Media, 2009.

7. P. Strzelecki: Analytical method for determining fatigue properties of materials and construction elements in high cycle life (in Polish), Uniwersytet TechnologicznoPrzyrodniczy w Bydgoszczy, 2014.

8. P. Strzelecki, J. Sempruch, K. Nowicki: Accuracy of Analytical-Experimental Method for Determining the Fatigue Characteristics in a Limited Life Region, Solid State Phenom. 224 (2014) 63-68. doi:10.4028/www.scientific. net/SSP.224.63.

9. T. Tomaszewski, J. Sempruch: Analysis of size effect in high-cycle fatigue for EN AW-6063, Solid State Phenom. 224 (2015) 75-80. doi:10.4028/www.scientific.net/SSP.224.75.

10. PN-H-04325:1976: Fatigue tests of metals -- Basic definition and general guidelines for the preparation of samples and tests, 1976.

11. P. Strzelecki, J. Sempruch: Experimental Verification of the Analytical Method for Estimated S-N Curve in Limited Fatigue Life, Mater. Sci. Forum. 726 (2012) 11-16. doi:10.4028/www.scientific.net/MSF.726.11.

12. ASTM E-739-91: Standard Practice for Statistical Analysis of Linear or Linearized Stress-Life (S-N) and Strain-Life Fatigue Data, 2006.

13. PN-EN-3987: Aerospace series - Test methods for metallic materials - Constant amplitude force-controlled high cycle fatigue testing, 2010.

14. ISO-12107: Metallic materials - fatigue testing - statistical planning and analysis of data, 2003.

15. F.G. Pascual, W.Q. Meeker: Estimating Fatigue Curves with the Random Fatigue-Limit Model, Technometrics. 41 (1999) 277-290. doi:10.2307/1271342.

16. S. Lorén, M. Lundström: Modelling curved S-N curves, Fatigue Fract. Eng. Mater. Struct. 28 (2005) 437-443. doi:10.1111/j.1460-2695.2005.00876.x.

17. R Core Team: $R$ : A language and environment for statistical computing, (2015). http://www.r-project.org/. 


\section{CONTACT WITH THE AUTHOR}

Paweł Strzelecki

Janusz Sempruch

University of Science and Technology in Bydgoszcz

Al. Prof. S. Kaliskiego 7

85-796 Bydgoszcz

Poland 